On a class of rotating gravitational waves

This article has been downloaded from IOPscience. Please scroll down to see the full text article.

2000 Class. Quantum Grav. 17533

(http://iopscience.iop.org/0264-9381/17/3/302)

View the table of contents for this issue, or go to the journal homepage for more

Download details:

IP Address: 128.206.162.204

The article was downloaded on $27 / 09 / 2010$ at $14: 57$

Please note that terms and conditions apply. 


\title{
On a class of rotating gravitational waves
}

\author{
Bahram Mashhoon $†$ James C McClune $\dagger$ and Hernando Quevedo $\ddagger$ \\ $\dagger$ Department of Physics and Astronomy, University of Missouri at Columbia, Columbia, \\ MO 65211, USA \\ ¥Instituto de Ciencias Nucleares, Universidad Nacional Autónoma de México, AP 70-543, \\ 04510 México DF, Mexico
}

Received 14 July 1999, in final form 11 October 1999

\begin{abstract}
A class of solutions of the gravitational field equations describing vacuum spacetimes outside rotating cylindrical sources is presented. The spacetime metric for this class is given by equation (35); to render this metric explicit, one must solve the nonlinear differential equation (31). A subclass of these solutions could correspond to the exterior gravitational fields of rotating cylindrical systems that emit gravitational radiation. This class has a special solutioncorresponding to the exact solution (32) of equation (31) - in common with the Robinson-Trautman gravitational wave spacetimes, namely, the Siklos solution. The properties of rotating gravitational waves are briefly investigated. In particular, we discuss the energy density of these waves using the gravitational stress-energy tensor.
\end{abstract}

PACS numbers: 0420,0430

\section{Introduction}

Rotating cylindrically symmetric gravitational waves were first discussed in $1990[1,2] \dagger$. The investigation of these solutions was motivated by Ardavan's discovery of the speed-of-light catastrophe [3] and its implications concerning gravitation [4]. Previous work is generalized in the present paper and an extended class of rotating gravitational wave spacetimes is analysed. By a rotating wave we mean radiation that propagates outward or inward and at the same time has non-trivial azimuthal motion. We find that the solution investigated previously $[1,2]$ is special, since it is the only member of the extended class studied here that represents the propagation of free rotating gravitational waves; moreover, we show that this special solution is locally equivalent to a Robinson-Trautman solution studied by Kerr and Debney and later on by Siklos.

The gravitational fields under consideration here are given by Ricci-flat spacetimes that are characterized by a metric of the form

$$
-\mathrm{d} s^{2}=\mathrm{e}^{2 \gamma-2 \psi}\left(-\mathrm{d} t^{2}+\mathrm{d} \rho^{2}\right)+\mu^{2} \mathrm{e}^{-2 \psi}(\omega \mathrm{d} t+\mathrm{d} \phi)^{2}+\mathrm{e}^{2 \psi} \mathrm{d} z^{2},
$$

in cylindrical coordinates $(\rho, \phi, z)$. Here $\gamma, \mu, \psi$ and $\omega$ are functions of $t$ and $\rho$ only; moreover, the signature of the spacetime metric is +2 throughout this paper and the speed of light in vacuum is set equal to unity. The gravitational field equations for the metric form (1) are given

$\dagger$ There is an error on p 289 of [2] in the discussion of the exterior field of a rotating cylinder. Contrary to the statement in the paper, the exterior vacuum field is not always static. It is, in fact, stationary for high angular momentum. This has been shown by Bonnor W B 1980 J. Phys. A: Math. Gen. 13 2121. BM is grateful to Professor Bonnor for clarifying remarks. Moreover, a typographical error occurs in equation (9): $\mu_{, u v}$ should be replaced by $\mu_{, v v}$. 
in appendix A. The physical motivations as well as a detailed discussion of certain aspects of the field equations for metric (1) are contained in previous papers [1,2], which should be consulted for further background information. Briefly, ansatz (1) is obtained from the general metric of rotating cylindrical gravitational waves with a constant linear polarization state by placing a certain restriction on the metric coefficients in order to reduce the number of unknown functions to four [2]. The spacetime represented by equation (1) admits two commuting spacelike Killing vectors $\partial_{z}$ and $\partial_{\phi}$. Though $\partial_{z}$ is hypersurface-orthogonal, $\partial_{\phi}$ is not; moreover, the isometry group is not orthogonally transitive. Vacuum solutions with two commuting Killing vector fields without the assumption of orthogonal transitivity have been considered by Gaffet [5]; however, it turns out that Gaffet's formalism is not directly applicable to our metric (1), since it is not of the form (1.6) of [5]. To retain our physical conceptions in this case $[1,2]$, we prefer to work with the metric in the form (1).

Instead of the variables $t$ and $\rho$, it is convenient to express the gravitational field equations in terms of the retarded and advanced times $u=t-\rho$ and $v=t+\rho$, respectively. The gravitational potentials for rotating waves are given by

$$
\begin{aligned}
& \left(\mu \psi_{v}\right)_{u}+\left(\mu \psi_{u}\right)_{v}=0, \\
& \mu_{u v}-\frac{1}{8} l^{2} \mu^{-3} \mathrm{e}^{2 \gamma}=0, \\
& \omega_{v}-\omega_{u}=l \mu^{-3} \mathrm{e}^{2 \gamma}, \\
& \gamma_{u}=\frac{1}{2 \mu_{u}}\left(\mu_{u u}+2 \mu \psi_{u}^{2}\right), \\
& \gamma_{v}=\frac{1}{2 \mu_{v}}\left(\mu_{v v}+2 \mu \psi_{v}^{2}\right),
\end{aligned}
$$

where $\psi_{u}=\partial \psi / \partial u$, etc. Here, $l$ is a constant length characteristic of the rotation of the system. We assume that $l>0$ throughout this paper that is specifically devoted to rotating gravitational waves. In fact, for $l=0$ the waves are non-rotating but expressed in a rotating frame of reference, since equation (4) implies that $\omega=\omega(t)$ in this case (cf appendix A). The explicit connection between $l$ and the rotation of the waves has been determined via a perturbative treatment in a previous work [2]. Using equation (3), it is possible to eliminate $\gamma$ from equations (5) and (6); then, we obtain the following equations:

$$
\begin{aligned}
& 2 \mu^{2} \psi_{u}^{2}=3 \mu_{u}^{2}-\mu \mu_{u u}+\mu \mu_{u} \mu_{u u v}\left(\mu_{u v}\right)^{-1}, \\
& 2 \mu^{2} \psi_{v}^{2}=3 \mu_{v}^{2}-\mu \mu_{v v}+\mu \mu_{v} \mu_{u v v}\left(\mu_{u v}\right)^{-1} .
\end{aligned}
$$

The integrability condition for this system, i.e. $\psi_{u v}=\psi_{v u}$, results in a nonlinear fourth-order partial differential equation for $\mu$. Alternatively, one could obtain the same equation for $\mu$ by combining equations (2), (7) and (8), which shows the consistency of the field equations (2)(6). It is important to notice that $\mu$ cannot be a function of $u$ or $v$ alone, since this possibility would be inconsistent with equation (3). The partial differential equation of fourth order for $\mu$ is, however, identically satisfied if $\mu$ is a separable function, i.e. $\mu=\alpha(u) \beta(v)$; this leads, in fact, to the rotating waves discussed earlier $[1,2]$. Here we wish to study a more general solution of the field equations.

In section 2, we discuss the solution of the field equations (2)-(6) for $l \neq 0$. We find that there are two possible classes of solutions. The first class corresponds to known solutions such as the stationary exterior field of a rotating cylindrical source, while the second class appears to represent a mixed situation involving rotating gravitational waves. In fact, a subclass of the latter solutions describes the exterior fields of certain rotating sources that emit gravitational radiation; these solutions approach the special rotating wave solution $[1,2]$ 
far from their sources. Indeed, the only solution of the second class corresponding to a pure gravitational wave spacetime is the special solution [1,2] that is a Robinson-Trautman solution (cf appendix B) and is further discussed in section 3. In section 4, some aspects of the energy, momentum and pressure of the rotating gravitational waves are discussed using the Bel-Robinson tensor [6]. Section 5 contains concluding remarks. The appendices contain some of the detailed calculations.

\section{Solution of the field equations}

To solve the field equations (2)-(6), let us introduce the functions $U, V$ and $W$ by

$$
U=\mu_{u} \gamma_{u}-\frac{1}{2} \mu_{u u}, \quad V=\mu_{v} \gamma_{v}-\frac{1}{2} \mu_{v v}, \quad W=\mu \gamma_{u v}+\frac{3}{2} \mu_{u v},
$$

and rewrite equations (5) and (6) as

$$
\mu \psi_{u}=\epsilon(\mu U)^{1 / 2}, \quad \mu \psi_{v}=\hat{\epsilon}(\mu V)^{1 / 2},
$$

where the symbols $\epsilon$ and $\hat{\epsilon}$ represent either +1 or -1 (i.e. $\epsilon^{2}=\hat{\epsilon}^{2}=1$ ). Using equation (3), it is straightforward to show that

$$
\mu U_{v}=\mu_{u} W, \quad \mu V_{u}=\mu_{v} W .
$$

Let us now combine relations (10) and (11) in order to satisfy equation (2); the result is

$$
\epsilon U^{-1 / 2}\left(\mu_{v} U+\mu_{u} W\right)+\hat{\epsilon} V^{-1 / 2}\left(\mu_{u} V+\mu_{v} W\right)=0 .
$$

This equation can be written as

$$
U\left(\mu_{u} V+\mu_{v} W\right)^{2}=V\left(\mu_{v} U+\mu_{u} W\right)^{2},
$$

which holds if either $W^{2}=U V$ or $\mu_{u}^{2} V=\mu_{v}^{2} U$; that is, equation (13) can be factorized into a fourth- and third-order equation, respectively.

Let us first consider the fourth-order equation $W^{2}=U V$. It follows from this relation and equation (10) that $W=\mp \mu \psi_{u} \psi_{v}$. The relation $W=-\mu \psi_{u} \psi_{v}$ is discussed in appendix A; indeed, it is a redundant field equation. In view of this fact, the other possibility $W=\mu \psi_{u} \psi_{v}$ simply implies $\psi_{u} \psi_{v}=0$; alternatively, this expression for $W$ along with $U=\mu \psi_{u}^{2}$ and $V=\mu \psi_{v}^{2}$ can be inserted into equation (11) to obtain relations that when combined with equation (2) in the form $2 \mu \psi_{u v}=-\left(\mu_{u} \psi_{v}+\mu_{v} \psi_{u}\right)$ imply $\psi_{u} \psi_{v}=0$. If $\psi$ is a function of either $u$ or $v$ alone, then equation (2) implies that $\psi$ must be a constant. The spacetime represented by a constant $\psi$ turns out to be a common special case of the solutions discussed in this section and is flat. Let us briefly digress here to discuss a subtle point regarding our approach. In this paper we attempt to derive the general solution of the field equations as revealed by our method. In this way we hope to find the general solution of the field equations, but there is no guarantee. In fact, the redundancy noted above may indicate a shortcoming of our approach in this respect. The resolution of this difficulty is beyond the scope of this paper.

Let us next consider $\mu_{u}^{2} V=\mu_{v}^{2} U$. It follows from equation (3) that

$$
\gamma_{u}=\frac{3}{2} \frac{\mu_{u}}{\mu}+\frac{1}{2} \frac{\mu_{u v u}}{\mu_{u v}}, \quad \gamma_{v}=\frac{3}{2} \frac{\mu_{v}}{\mu}+\frac{1}{2} \frac{\mu_{u v v}}{\mu_{u v}},
$$

which together with equations (9) imply that $\mu_{u}^{2} V=\mu_{v}^{2} U$ is essentially equivalent to

$$
\frac{\mu_{u v v}}{\mu_{v}}-\frac{\mu_{v v} \mu_{u v}}{\mu_{v}^{2}}=\frac{\mu_{u v u}}{\mu_{u}}-\frac{\mu_{u u} \mu_{u v}}{\mu_{u}^{2}} .
$$

Equation (15) can be written as $\left(\ln \mu_{v}\right)_{u v}=\left(\ln \mu_{u}\right)_{v u}$. Let $f(u)$ and $g(v)$ be arbitrary functions of their arguments and consider the transformation $(u, v) \rightarrow(x, y)$ such that

$$
x=f(u)+g(v), \quad y=f(u)-g(v),
$$


$f_{u} \neq 0$, and $g_{v} \neq 0$; then, the general solution for $\mu$ is that $\mu=\mu(x)$, i.e. $\mu$ should not depend on $y$.

Let us now proceed to the calculation of $\psi$. To this end, let us consider a function $\mathcal{L}(x)$ given by

$$
\mathcal{L}^{2}(x)=\frac{1}{2}\left(3 \mu^{\prime 2}-\mu \mu^{\prime \prime}+\mu \frac{\mu^{\prime} \mu^{\prime \prime \prime}}{\mu^{\prime \prime}}\right),
$$

where a prime indicates differentiation with respect to $x$. Equations (7) and (8) can now be written as

$$
\mu^{2} \psi_{u}^{2}=f_{u}^{2} \mathcal{L}^{2}, \quad \mu^{2} \psi_{v}^{2}=g_{v}^{2} \mathcal{L}^{2} .
$$

Furthermore,

$$
\psi_{u}=\left(\frac{\partial \psi}{\partial x}+\frac{\partial \psi}{\partial y}\right) f_{u}, \quad \psi_{v}=\left(\frac{\partial \psi}{\partial x}-\frac{\partial \psi}{\partial y}\right) g_{v} .
$$

Combining equations (18) and (19), we find that either $\psi$ is purely a function of $x$ given by $\mu^{2}(\mathrm{~d} \psi / \mathrm{d} x)^{2}=\mathcal{L}^{2}(x)$ or $\psi$ is purely a function of $y$ given by $\mu^{2}(\mathrm{~d} \psi / \mathrm{d} y)^{2}=\mathcal{L}^{2}(x)$. These cases will now be discussed in turn.

\subsection{Case (i): $\psi=\psi(x)$}

It follows from equation (2) that in this case

$$
\frac{\mathrm{d} \psi}{\mathrm{d} x}=\frac{C}{\mu(x)},
$$

where $C$ is an integration constant. Thus $\mu(x)$ is determined by $\mathcal{L}^{2}(x)=C^{2}$. To solve this differential equation, let $X=\mu^{\prime}$ and note that

$$
\mathcal{L}^{2}=\frac{1}{2} X^{2}\left(\mu^{2} \frac{\mathrm{d} X}{\mathrm{~d} \mu}\right)^{-1} \frac{\mathrm{d}}{\mathrm{d} \mu}\left(\mu^{3} \frac{\mathrm{d} X}{\mathrm{~d} \mu}\right) .
$$

Moreover, let $S=\mu \mathrm{d} X / \mathrm{d} \mu$; then, $\mathcal{L}^{2}(x)=C^{2}$ can be written as

$$
\frac{\mathrm{d} S}{\mathrm{~d} X}=2\left(\frac{C^{2}}{X^{2}}-1\right),
$$

which can be integrated to give $S=-2\left(D+X+C^{2} / X\right)$. Here $D$ is an integration constant. It follows from this result that $\mu(x)$ can be found implicitly from $\mathrm{d} \mu=X(\mu) \mathrm{d} x$, where

$$
\mu^{2}=\exp \left(-\int \frac{X \mathrm{~d} X}{X^{2}+D X+C^{2}}\right) .
$$

The spacetime metric in this case can be written in a form that depends only on $x$. That is, it is possible to show—by a transformation of the metric to normal form - that the general solution in case (i) has an extra timelike or spacelike Killing vector field $\partial_{y}$. To this end, let us consider the coordinate transformation $(t, \rho, \phi, z) \rightarrow(T, R, \Phi, Z)$, where $f(u)=(T+R) / 2$, $g(v)=(-T+R) / 2, \phi=\Phi+H(T, R)$ and $z=Z$. In this transformation $H(T, R)$ is a solution of the partial differential equation

$$
-(F+G) \frac{\partial H}{\partial T}+(F-G) \frac{\partial H}{\partial R}+\frac{2}{l}(F+G) \frac{\mathrm{d} \mu}{\mathrm{d} R}=0,
$$

where $F=f_{u}^{-1}, G=g_{v}^{-1}, R=x$ and $T=y$. To prove our assertion, we will show that under this coordinate transformation the spacetime metric in case (i) takes the form

$-\mathrm{d} s^{2}=P(R)\left(-\mathrm{d} T^{2}+\mathrm{d} R^{2}\right)+\mu^{2}(R) \mathrm{e}^{-2 \psi(R)}[\Omega(R) \mathrm{d} T+\mathrm{d} \Phi]^{2}+\mathrm{e}^{2 \psi(R)} \mathrm{d} Z^{2}$, 
which is clearly invariant under a translation in $T$ thus implying the existence of a Killing vector field $\partial_{T}$. It follows from a comparison of the metric forms (1) and (25) that

$$
\begin{aligned}
& P(R)=-\frac{2}{l^{2}} \mu^{3} \frac{\mathrm{d}^{2} \mu}{\mathrm{d} R^{2}} \mathrm{e}^{-2 \psi(R)}, \\
& \frac{\partial H}{\partial T}=-\frac{1}{4} \omega(F-G)+\Omega(R), \\
& \frac{\partial H}{\partial R}=-\frac{1}{4} \omega(F+G) .
\end{aligned}
$$

Equations (27) and (28) can be used in equation (24) to show that $\Omega(R)=2 l^{-1} \mathrm{~d} \mu / \mathrm{d} R$. It remains to show that the integrability condition for equations (27) and (28), i.e. $\partial^{2} H / \partial R \partial T=$ $\partial^{2} H / \partial T \partial R$, is satisfied. It turns out that this relation is indeed true, since it is equivalent to the field equation (4) for $\omega$.

Let us note that the solution of equation (22) implies that in case (i)

$$
\frac{\mathrm{d}^{2} \mu}{\mathrm{d} R^{2}}=-\frac{2}{\mu}\left[\left(\frac{\mathrm{d} \mu}{\mathrm{d} R}\right)^{2}+D \frac{\mathrm{d} \mu}{\mathrm{d} R}+C^{2}\right],
$$

and one can show explicitly that the metric form (25) is flat once $C=0$. For $C \neq 0$, the general solution of case (i) is not flat. If $P(R)>0$, a simple redefinition of the radial coordinate can be used to cast equation (25) in Lewis form, i.e. solution (25) is a member of the class of exterior stationary solutions found by Lewis (see [7], ch 20). For $P(R)<0$, the solution contains three commuting spacelike Killing vectors and belongs to the family of Kasner solutions (see [7], ch 11).

\subsection{Case (ii): $\psi=\psi(y)$}

It follows from $(\mathrm{d} \psi / \mathrm{d} y)^{2}=\mathcal{L}^{2}(x) / \mu^{2}$ that $\psi$ must be a linear function of $y$, since the left-hand side of this equation is purely a function of $y$, while the right-hand side is purely a function of $x$; therefore, each side must be constant. Thus $\psi=a y+b$, where $a$ and $b$ are constants, and $\mathcal{L}^{2}=a^{2} \mu^{2}$. It turns out that equation (3) is identically satisfied in this case. Using equation (21), the differential equation for $\mu$ can be expressed in terms of $X=\mu^{\prime}$ as

$$
X^{2} \frac{\mathrm{d}}{\mathrm{d} \mu}\left(\mu^{3} \frac{\mathrm{d} X}{\mathrm{~d} \mu}\right)=2 a^{2} \mu^{4} \frac{\mathrm{d} X}{\mathrm{~d} \mu} .
$$

If $a=0, \psi$ is constant and we recover the same flat spacetime solution as in case (i) with $C=0$. Therefore, let $a \neq 0$ and consider a new 'radial' coordinate $r$ given by $r=\left(a^{2} \mu^{2}\right)^{-1}$; then,

$$
r^{2} X^{2} \frac{\mathrm{d}^{2} X}{\mathrm{~d} r^{2}}+\frac{\mathrm{d} X}{\mathrm{~d} r}=0 .
$$

The solution $X=$ constant is unacceptable, since it implies that $\gamma=-\infty$. However, there is another exact solution that is given by

$$
X= \pm\left(\frac{3}{2} r\right)^{-1 / 2}
$$

which turns out to correspond to the special rotating gravitational waves $[1,2]$ that are the subject of the next section. It is possible to transform equation (31) to an autonomous form; to this end, let us define $\delta$ and $\xi$ such that $\delta=2 /\left(3 r X^{2}\right)$ and $\xi=-4 X_{r} /\left(3 X^{3}\right)$, where $X_{r}=\mathrm{d} X / \mathrm{d} r$. It can then be shown that equation (31) is equivalent to

$$
\frac{\mathrm{d} \xi}{\mathrm{d} \delta}=\frac{3}{2} \frac{\xi\left(\xi-\delta^{2}\right)}{\delta(\xi-\delta)}
$$




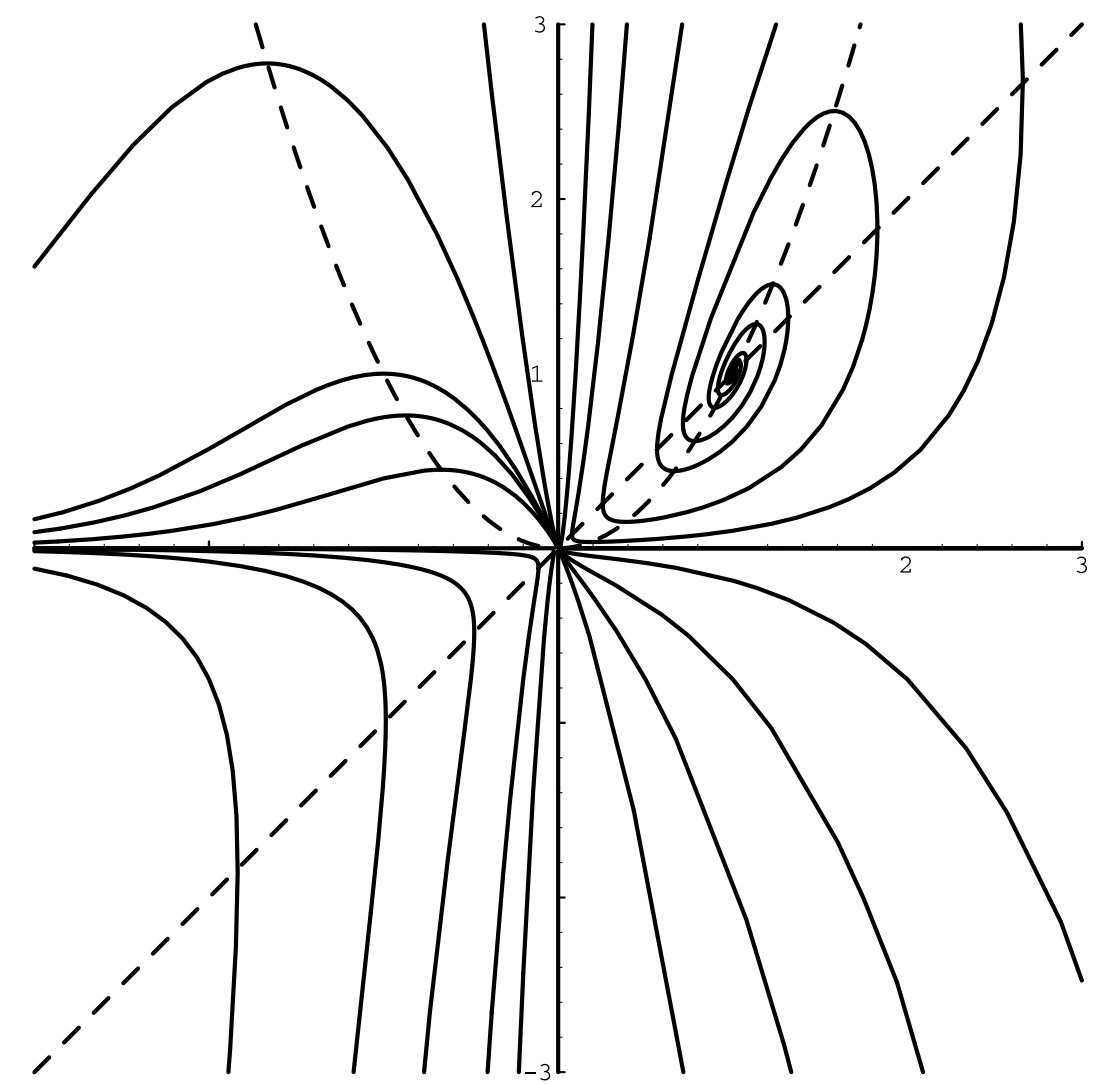

Figure 1. The plot of $\xi$ versus $\delta$ for equation (33) illustrating the spiral structure near the singular point $(1,1)$ corresponding to the special solution (32). In fact, the plot displays some of the characteristic curves of the autonomous system $\mathrm{d} \delta / \mathrm{d} \theta=2 \delta(\xi-\delta)$ and $\mathrm{d} \xi / \mathrm{d} \theta=3 \xi\left(\xi-\delta^{2}\right)$. The $\delta$ and $\xi$ nullclines are represented by broken curves $\delta=\xi$ and $\xi=\delta^{2}$, respectively. The critical point $(0,0)$ is a degenerate singularity of the system, while the other critical point $(1,1)$ is a simple singularity.

where $(\delta, \xi)=(1,1)$ represents the special solution (32). This special solution is an isolated singularity of the nonlinear autonomous system (33) and the behaviour of characteristics near this point indicates that $(1,1)$ is a spiral point as shown in figure 1 .

Let us note here certain general features of $X(r)$, which is the solution of equation (31). If $X(r)$ is a solution, then so is $-X(r)$. Moreover, equation (31) can be written as $\left(X_{r}^{2}\right)_{r}=-2 X_{r}^{2} /\left(r^{2} X^{2}\right)$, which indicates that for $X \neq$ constant the absolute magnitude of the slope of $X(r)$ decreases monotonically as $r$ increases. If $X(r)$ has a zero at $r_{0} \neq 0$, then the behaviour of $X(r)$ near $r_{0}$ is given by

$$
X(r)= \pm \sqrt{\frac{2}{r_{0}}}\left(\frac{r}{r_{0}}-1\right)^{1 / 2}\left[1+\frac{1}{2}\left(\frac{r}{r_{0}}-1\right)-\frac{3}{76}\left(\frac{r}{r_{0}}-1\right)^{2}+\cdots\right]
$$

for $r \geqslant r_{0}$. These results are illustrated in figure 2 .

Once a solution $X(r)$ of equation (31) is given, one can find a solution of the field equations in case (ii). It turns out that in general such a solution is not a pure gravitational wave. The only exception is the special solution (32). To demonstrate this, let us first consider a transformation of the spacetime metric (1) to the normal form appropriate for 


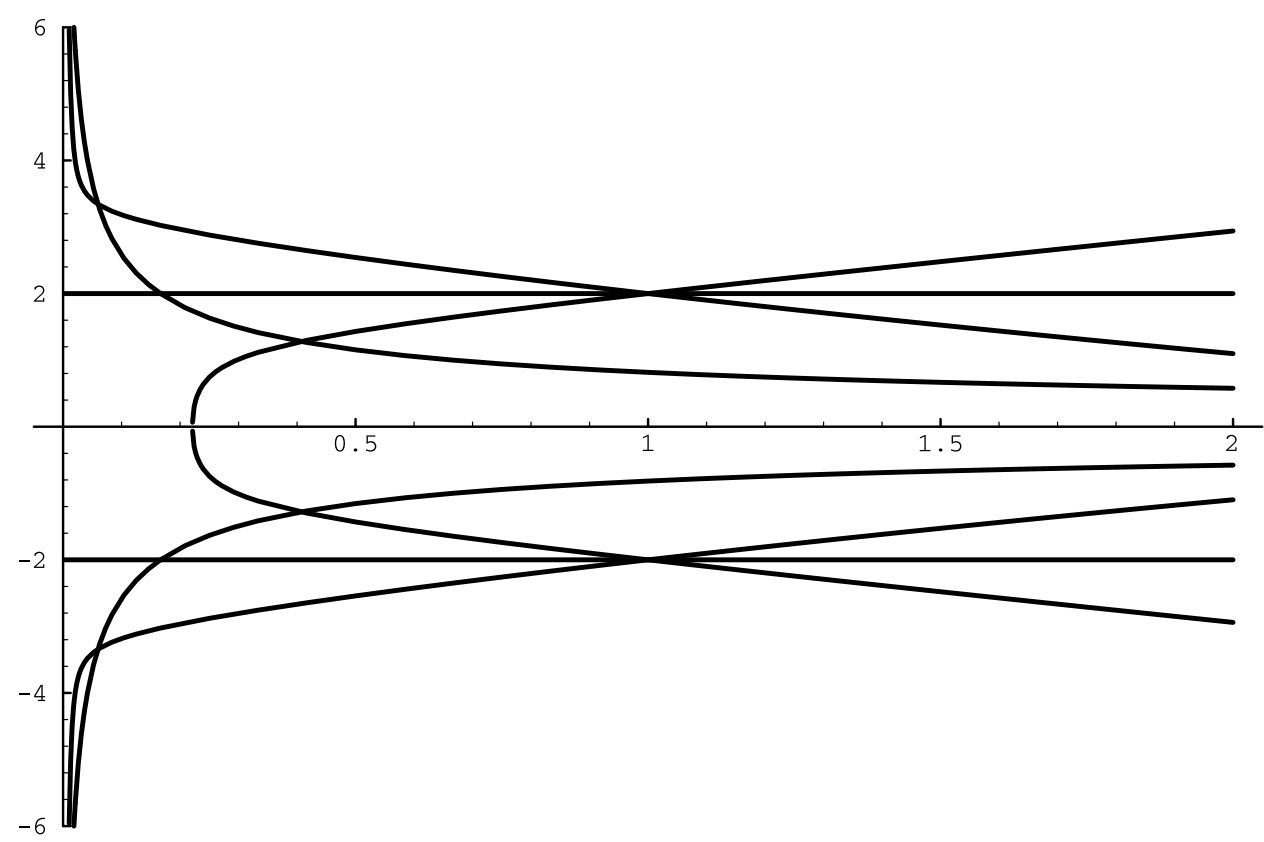

Figure 2. The plot of $X(r)$ versus $r$ for three solutions of equation (31) with the boundary conditions that at $r=1, X=2$ and $X_{r}=-1,0,1$. The function $-X(r)$ is plotted in this figure as well. Note that the solution for $X_{r}(r=1)=-1$ approaches the special solution (32), also depicted here, for $r \rightarrow 0$. The behaviour of the solution for $X_{r}(r=1)=1$ near $r_{0} \simeq 0.22$ is in accordance with equation (34). These results should be compared with the first and fourth quadrants of the $(\delta, \xi)$-plane in figure 1.

case (ii). The first step in this transformation is exactly the same as that given in case (i) and equation (24), i.e. $(t, \rho, \phi, z) \rightarrow(T, R, \Phi, Z)$. Next, we change the radial coordinate to $r$ and let $(T, R, \Phi, Z) \rightarrow(\hat{t}, r, \Phi, \hat{Z})$, where $\hat{t}=2 a T$ and $\hat{Z}=l a^{2} \exp (2 b) Z$. In terms of the new system of coordinates, the spacetime metric may be expressed-up to an overall constant factor-as

$$
-\mathrm{d} s^{2}=\mathrm{e}^{-\hat{t}} \frac{X_{r}}{X}\left(-X^{2} \mathrm{~d} \hat{t}^{2}+\frac{\mathrm{d} r^{2}}{r^{3}}\right)+\frac{\mathrm{e}^{-\hat{t}}}{\hat{l}^{2} r}(\hat{l} X \mathrm{~d} \hat{t}+\mathrm{d} \Phi)^{2}+\mathrm{e}^{\hat{t}} \mathrm{~d} \hat{Z}^{2},
$$

where $\hat{l}=(l a)^{-1}$ is a constant and $X(r)$ is a solution of equation (31). There are four curvature invariants for this Ricci-flat spacetime and it is possible to show that two of these are identically zero. The other two also vanish for the special solution (32); therefore, this special case corresponds to free gravitational waves [8]. Specifically, let

$$
\begin{aligned}
& I_{1}=R_{\mu \nu \rho \sigma} R^{\mu \nu \rho \sigma}-\mathrm{i} R_{\mu \nu \rho \sigma} R^{* \mu \nu \rho \sigma}, \\
& I_{2}=R_{\mu \nu \rho \sigma} R^{\rho \sigma \alpha \beta} R_{\alpha \beta}{ }^{\mu \nu}+\mathrm{i} R_{\mu \nu \rho \sigma} R^{\rho \sigma \alpha \beta} R_{\alpha \beta}^{*}{ }^{\mu \nu},
\end{aligned}
$$

be the complex invariants of spacetime. We find that for the metric form (35) these are real and are given by

$$
\begin{aligned}
& I_{1}=-\mathrm{e}^{2 \hat{t}}\left(r X^{4} X_{r}^{2}\right)^{-1}\left(1-3 r X^{2}-2 r^{2} X X_{r}-r^{3} X^{3} X_{r}+r^{4} X^{2} X_{r}^{2}\right), \\
& I_{2}=-\frac{3}{4} \mathrm{e}^{3 \hat{t}}\left(r X^{5} X_{r}^{3}\right)^{-1}\left(1-2 r X^{2}-2 r^{2} X X_{r}+2 r^{3} X^{3} X_{r}+r^{4} X^{2} X_{r}^{2}\right) .
\end{aligned}
$$

It is important to note that $I_{1}$ and $I_{2}$ vanish for the special solution $X= \pm(3 r / 2)^{-1 / 2}$, indicating that it describes the propagation of free gravitational waves. Moreover, all other solutions- 
which do not describe free gravitational waves—are singular for $\hat{t} \rightarrow \infty$, since $I_{1}$ and $I_{2}$ both diverge in the infinite 'future'. Thus all such solutions 'evolve' to states that are ultimately singular. The singular nature of the special solution (32) has been discussed previously [1,2]. It is important to point out that for some solutions $X(r)$ of equation (31), $r$ is in fact the temporal coordinate in the spacetime metric (35). One can show that $\hat{t}$ satisfies the scalar wave equation, $g^{\mu \nu} \Psi_{; \mu \nu}=0$, in the spacetime given by the metric form (35); therefore, $\hat{t}$ must in general be interpreted as the scalar potential for the cylindrical gravitational waves. Indeed, $\exp (\hat{t} / 2)$ is the scalar magnitude of the spacelike Killing vector associated with translational invariance along the axis of cylindrical symmetry.

It is clear from equation (31) and figure 2 that as $r \rightarrow 0, X(r) \rightarrow \pm(3 r / 2)^{-1 / 2}$ for a subclass of the spacetimes under consideration here; indeed, it is simple to check that $I_{1} \rightarrow 0$ and $I_{2} \rightarrow 0$ as $r \rightarrow 0$ for the solution given in figure 2 with $X_{r}(r=1)=-1$. Moreover, $I_{1}$ and $I_{2}$ diverge as $r \rightarrow \infty$. In fact, inspection of the first quadrant of figure 1 shows that these properties are shared by many solutions with $X_{r} / X<0$. We might expect that such solutions correspond to the exterior field of a rotating and radiating cylindrical source such that very far from the symmetry axis the metric would nearly describe free rotating gravitational waves given by the unique special solution (32); of course, one would need to find an appropriate interior solution for the source. In this case, it is useful to introduce a new radial coordinate $\tilde{R}=r^{-1 / 2} \exp (-\hat{t} / 2)$, which is the magnitude of the Killing vector $\partial_{\Phi}$ up to a proportionality constant. Thus $r \rightarrow 0$ or $\hat{t} \rightarrow-\infty$ corresponds to $\tilde{R} \rightarrow \infty$, so that far away from the symmetry axis $I_{1} \rightarrow 0$ and $I_{2} \rightarrow 0$, while for $r \rightarrow \infty$ or $\hat{t} \rightarrow \infty, \tilde{R} \rightarrow 0$ and $I_{1}$ and $I_{2}$ are both divergent. It follows that the axis of cylindrical symmetry is always singular and that far from the axis the spacetime approaches the special free rotating wave solution that has singularities and is discussed in the next section. For a singular axis, the definition of axisymmetry is problematic [9]; hence, difficulties may arise in interpreting these solutions as rotating cylindrical gravitational waves. Such radiation is presumably emitted by a regular rotating cylindrical source; the waves then propagate outward to become essentially free rotating waves far from the source. A detailed interpretation as well as the matching of the exterior solution with an interior solution is beyond the scope of this work.

The general rotating wave solution (35) turns out to be of type I in the Petrov classification. Thus rotating gravitational wave spacetimes are algebraically general except in the case of the special solution (32), which is algebraically special and of Petrov type III. This solution turns out to be locally equivalent to a special Robinson-Trautman solution as shown in appendix B. The Robinson-Trautman class of gravitational wave spacetimes only involves algebraically special solutions and is therefore distinct from the class of rotating wave spacetimes presented in this paper. It is interesting to note that the Robinson-Trautman class can be simply generalized by the inclusion of a cosmological constant $\Lambda$ (see [7], ch 24). Thus the special rotating wave solution has a $\Lambda \neq 0$ generalization studied by Siklos (see equation (11.45) on p 134 of [7]). Further investigation is needed to determine whether the class of rotating gravitational waves can be generalized to include a cosmological constant.

The class of rotating gravitational waves is given by the metric form (35), where $X(r)$ is a solution of the nonlinear differential equation (31). This equation remains invariant under the scale transformation $\sigma:(r, X) \rightarrow\left(\sigma^{2} r, \sigma^{-1} X\right)$, where $\sigma$ is a constant. The spacetime (35) involving a solution of (31) that also remains invariant under such scaling — such as the special solution (32) — would then contain a third Killing vector. In fact, inspection of the metric form (35) reveals that it remains unchanged to first order in $\epsilon_{0}, 0<\epsilon_{0} \ll 1$, under the transformations $\hat{t} \rightarrow \hat{t}-2 \epsilon_{0}, r \rightarrow\left(1+\epsilon_{0}\right) r, X \rightarrow\left(1-\epsilon_{0} / 2\right) X, \Phi \rightarrow\left(1-\epsilon_{0} / 2\right) \Phi$ and $\hat{Z} \rightarrow\left(1+\epsilon_{0}\right) \hat{Z}$. This observation indicates the existence of a third Killing vector given by $\hat{K}^{\mu}=(-2, r,-\Phi / 2, \hat{Z})$; moreover, $\hat{K}^{\mu}$ is not hypersurface-orthogonal. The existence of this 
additional spacelike Killing vector for the special solution (32) does not extend to the general solution. The quantities $\delta$ and $\xi$ in equation (33) are invariant under the scale transformation $\sigma$; however, a general solution $X(r)$ of equation (31) is not. To our knowledge, only the special solution contains a three-parameter group of isometries. It is shown in appendix B that this solution is of type $\mathrm{VI}_{h}$, with $h=-\frac{1}{9}$, in the Bianchi classification and that it is locally the same as a special Robinson-Trautman solution. Thus the rotating vacuum solutions of the gravitational field equations for the metric form (1) in cases (i) and (ii) either contain an additional Killing vector field, in which case they are known [7], or they are given by the metric form (35), where $X(r) \neq \pm(3 r / 2)^{-1 / 2}$ must apparently be obtained numerically from equation (31). A complete numerical investigation of possible solutions given by equations (31) and (35) and partly revealed in figure 2 is beyond the scope of this paper. However, it is possible to work out some aspects of the energy density and pressure of the rotating gravitational waves. This subject is taken up in section 4 .

Finally, let us note that the unique special solution corresponding to free gravitational waves (32) can be written in terms of $\mu$ and $x$ as $\mathrm{d} \mu / \mathrm{d} x= \pm\left(\frac{2}{3}\right)^{1 / 2} a \mu$, which can be easily solved to show that $\mu$ depends exponentially upon $x=f(u)+g(v)$. This means simply that $\mu$ can be written as $\mu=\alpha(u) \beta(v)$, where $\alpha$ and $\beta$ are arbitrary functions. The next section is devoted to a discussion of the properties of this solution beyond what is already known from previous studies $[1,2]$.

\section{Free rotating gravitational waves}

Free non-rotating cylindrical gravitational wave solutions of Einstein's equations were first discussed by Beck [10]. These solutions have been the subject of many subsequent investigations $[8,11,12]$. The solutions considered in this section can be interpreted in terms of simple cylindrical waves that rotate [2], though they are all locally equivalent to a special Robinson-Trautman solution that has been investigated by Kerr and Debney and Siklos (cf appendix B).

The special solution (32) for free rotating gravitational waves is given by

$$
\begin{array}{ll}
\mu & =\alpha(u) \beta(v), \quad \psi=\sqrt{\frac{3}{2}} \ln \frac{\alpha}{\beta}, \\
\gamma & =\frac{1}{2} \ln \left[\frac{8}{l^{2}}(\alpha \beta)^{3} \alpha_{u} \beta_{v}\right], \quad \omega_{v}-\omega_{u}=\frac{8}{l} \alpha_{u} \beta_{v} .
\end{array}
$$

It turns out that in this case equation (2) is equivalent to the scalar wave equation for $\psi$ in the background geometry given by equation (1); therefore, the function $\psi$, which is a mixture of ingoing and outgoing waves according to equation (40), has the interpretation of the scalar potential for the free rotating gravitational waves. The solution (40) and (41) cannot be thought of as a collision between outgoing and ingoing gravitational waves, since the field equations do not admit solutions for which $\mu=\mu(u)$ or $\mu=\mu(v)$. That is, there is no purely outgoing solution just as there is no purely ingoing solution. The curvature invariants all vanish for the special solution (32); in fact, the free waves are of type III in the Petrov classification [1].

The spacetime given by equations (40) and (41) is singular. In fact, the analysis of the spacetime curvature indicates that moving singular cylinders appear whenever $\alpha, \beta, \alpha_{u}$ or $\beta_{v}$ vanishes. It is interesting to consider the nature of the symmetry axis for rotating waves, since in these solutions the axis does not, in general, satisfy the condition of elementary flatness. If for an infinitesimal spacelike circle around the axis of symmetry the ratio of circumference to radius goes to $2 \pi$ as the radius goes to zero, the condition of elementary flatness is satisfied for the axis under consideration. In our case, this means that $\mu^{2} /\left(\mathrm{e}^{2 \gamma} \rho^{2}\right) \rightarrow 1$ as $\rho \rightarrow 0$. 
For simple cylindrical waves (i.e. Beck's solution) we have $\mu=\rho$ and hence the condition of elementary flatness is that $\gamma \rightarrow 0$ as $\rho \rightarrow 0$. In general, Beck's fields can be divided into two classes: the Einstein-Rosen waves [11] and the Bonnor-Weber-Wheeler waves [12]. In the former class, the axis does not satisfy the condition of elementary flatness; in fact, the axis is not regular either. It is a singularity of spacetime and is therefore interpreted as the source of the cylindrical waves which are otherwise free of singularities. In the latter case, the axis does satisfy the condition of elementary flatness and is, moreover, regular. The waves presumably originate at infinity: incoming waves implode on the axis and then move out to infinity with no singularities in the finite regions of spacetime. It is therefore clear that no caustic cylinders appear regardless of the nature of the axis. They do appear, however, when the waves rotate. We are therefore led to regard the appearance of moving singular cylinders in our solution as being due to the rotation of the waves. It is important to emphasize the absence of a direct causal connection between the violation of elementary flatness at the axis and the presence of singular cylinders: the axis is static while the singularity is in motion, and the condition of elementary flatness involves the gravitational potentials $\left(g_{\mu \nu}\right)$, while the singularity of the field has to do with the spacetime curvature. In fact, it is possible to find instances of exact solutions for which the axis is elementary flat but singular [13]. A discussion of related issues is contained in [9].

In spacetime regions between the extrema of $\alpha$ and $\beta$, the solution (40) and (41) can be reduced to a normal form in which $\alpha$ and $\beta$ are linear functions of their arguments [1]. With further elementary coordinate transformations, the normal form can be reduced to two special solutions which are of interest: (a) $\alpha=\sigma_{0} u, \beta=\sigma_{0} v$ and (b) $\alpha=\sigma_{0} u, \beta=-\sigma_{0} v$. Here $\sigma_{0}= \pm\left(l_{0}\right)^{-1 / 2}$, where $l_{0}$ is a constant length whose introduction is necessary on dimensional grounds. The metrics for these cases are, respectively,

$-\mathrm{d} s^{2}=\frac{8(u v)^{3}}{l_{0}^{4} l^{2}}\left(\frac{u}{v}\right)^{-\sqrt{6}}\left(-\mathrm{d} t^{2}+\mathrm{d} \rho^{2}\right)+\frac{(u v)^{2}}{l_{0}^{2}}\left(\frac{u}{v}\right)^{-\sqrt{6}}\left(\frac{8 \rho}{l_{0} l} \mathrm{~d} t+\mathrm{d} \phi\right)^{2}+\left(\frac{u}{v}\right)^{\sqrt{6}} \mathrm{~d} z^{2}$

and

$-\mathrm{d} s^{2}=\frac{8(u v)^{3}}{l_{0}^{4} l^{2}}\left(-\frac{u}{v}\right)^{-\sqrt{6}}\left(-\mathrm{d} t^{2}+\mathrm{d} \rho^{2}\right)+\frac{(u v)^{2}}{l_{0}^{2}}\left(-\frac{u}{v}\right)^{-\sqrt{6}}\left(\frac{8 \rho}{l_{0} l} \mathrm{~d} t-\mathrm{d} \phi\right)^{2}+\left(-\frac{u}{v}\right)^{\sqrt{6}} \mathrm{~d} z^{2}$.

To ensure that the spacetime metric is real, case (a) must be limited to $(u>0, v>0)$ or $(u<0, v<0)$. Similarly, case (b) must be limited to $(u>0, v<0)$ or $(u<0, v>0)$. In either case the hypersurfaces $u=0$ and $v=0$ are curvature singularities. Since the laws of physics break down very close to these surfaces, it appears that the consideration of boundary conditions across such surfaces would be without physical significance. In case (b), $\rho$ is a temporal coordinate and $t$ is a spatial coordinate. The transformation $(t \rightarrow \rho, \rho \rightarrow t)$, or equivalently ( $u \rightarrow-u, v \rightarrow v$ ), brings the metric to a form which reduces to case (a) with a further coordinate transformation $\phi \rightarrow \phi+8 t \rho /\left(l_{0} l\right)$. Therefore, only the normal form in case (a) will be considered in the remainder of this paper.

The geodesic equation for free rotating gravitational wave spacetimes is discussed in appendix $\mathrm{C}$.

\section{The gravitational stress-energy tensor}

In our recent work [6], a fundamental pseudo-local gravitoelectromagnetic stress-energy tensor has been defined via a certain averaging procedure in a Fermi frame along the path of a geodesic observer. The result can then be pointwise extended to arbitrary observers, following the standard manner involving instantaneous measurements, in accordance with 
the basic assumption of locality in general relativity. For Ricci-flat spacetimes, the gravitoelectromagnetic stress-energy tensor becomes the gravitational stress-energy tensor $T_{\mu \nu}$ given by

$$
T_{\mu \nu}=\frac{L^{2}}{12 \pi G_{0}} T_{\mu \nu \rho \sigma} \lambda_{(0)}^{\rho} \lambda_{(0)}^{\sigma}=\frac{L^{2}}{12 \pi G_{0}} T_{\mu \nu(0)(0)},
$$

where $G_{0}$ is Newton's constant, $L$ is a constant length-scale characteristic of the field under consideration, $T_{\mu \nu \rho \sigma}$ is the Bel-Robinson tensor $[6,8,14]$ defined by

$$
T_{\mu \nu \rho \sigma}=\frac{1}{2}\left(R_{\mu \xi \rho \zeta} R_{\nu}^{\xi} \sigma^{\zeta}+R_{\mu \xi \sigma \zeta} R_{\nu}^{\xi} \rho^{\zeta}\right)-\frac{1}{16} g_{\mu \nu} g_{\rho \sigma} R_{\alpha \beta \gamma \delta} R^{\alpha \beta \gamma \delta},
$$

and $\lambda_{(0)}^{\mu}=\mathrm{d} x^{\mu} / \mathrm{d} \tau$ is the vector tangent to the timelike path of the observer. The gravitational stress-energy tensor is expected to provide approximate measures of the average gravitational energy, momentum and stress in the neighbourhood of the observer.

The result of the calculation of $T_{\mu \nu}$ for a simple class of linearly polarized plane gravitational waves is given in our 1997 paper [6]. A systematic study of $T_{\mu \nu}$ for gravitational radiation spacetimes (such as, for example, Beck's fields) would be a worthwhile endeavour. Here, however, we focus attention on rotating waves and present the results of certain relatively tractable calculations.

Let us imagine a spacetime given by the metric form (35), where $X(r)$ is a solution of equation (31) such that far from the symmetry axis it approaches the free rotating gravitational waves. As is evident from figure 2, for such solutions of equation (31), $X X_{r}<0$. Inspection of the metric form (35) then reveals that in this case $\hat{t}$ is a spatial coordinate and $r$ is the temporal coordinate. Consider now the class of static observers whose orthonormal tetrad frame, in $(\hat{t}, r, \Phi, \hat{Z})$ coordinates, consists of the temporal axis

$$
\left(-\mathrm{e}^{-\hat{t}} \frac{X_{r}}{r^{3} X}\right)^{-1 / 2}(0,1,0,0)
$$

as well as the spacelike unit vector $\exp (-\hat{t} / 2)(0,0,0,1)$ parallel to the axis of symmetry. The other two spatial axes will be ignored for the sake of simplicity. It can be shown that the energy density of the gravitational field according to the static observers is

$$
\rho_{g}=\left(\frac{L^{2}}{96 \pi G_{0}}\right) \frac{\mathrm{e}^{2 \hat{t}}}{X^{2} X_{r}^{2}}\left[1+\frac{1}{2 r X^{2}}\left(1-r^{2} X X_{r}\right)\left(1+r X^{2}-r^{2} X X_{r}\right)\right],
$$

while the flux of gravitational energy parallel to the axis of symmetry vanishes. However, the radiation has pressure along this direction given by

$$
p_{g}=\left(\frac{L^{2}}{96 \pi G_{0}}\right) \frac{\mathrm{e}^{2 \hat{t}}}{X^{2} X_{r}^{2}} .
$$

The gravitational energy density and pressure are positive and $p_{g}<\rho_{g}$. For the special exact solution (32) corresponding to free rotating waves, these results reduce to $\rho_{g}=3 p_{g}$ and $p_{g}=\left(3 L^{2} / 32 \pi G_{0}\right) r^{4} \exp (2 \hat{t})$; indeed, the partial equation of state $p_{g}=\rho_{g} / 3$ appears to be consistent with the interpretation of this solution in terms of free gravitational waves.

It would be interesting to study the energy of rotating gravitational waves along the worldline of a geodesic observer. However, the spacetime metric for the rotating waves (35) is given explicitly only for the case of the exact solution (32) of the differential equation (31). This unique solution is approached asymptotically by the rotating-wave solutions very far from the symmetry axis. For the sake of simplicity, we therefore restrict attention to the special solution (32) that, according to the results presented in section 3, corresponds to the free rotating waves. 


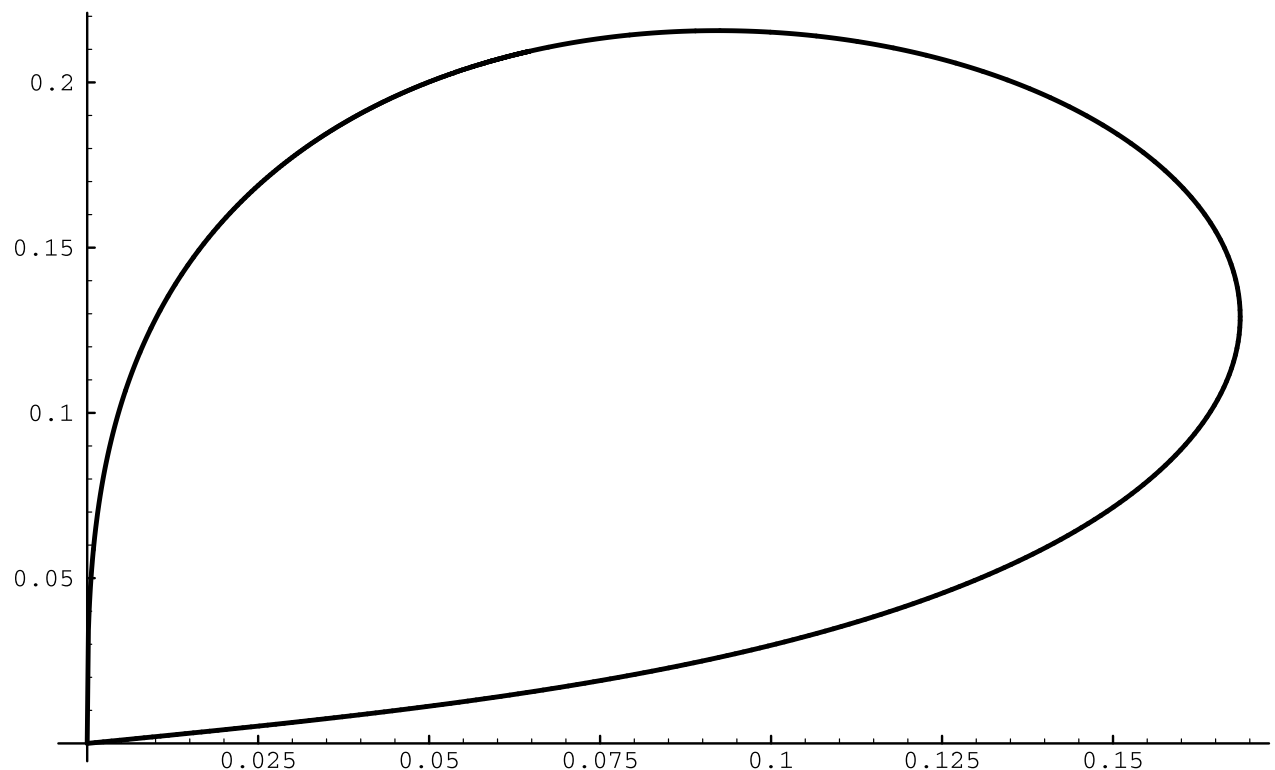

Figure 3. The functions $\rho(\tau) / l_{0}$ and $\phi(\tau)$ given by equations (47) and (48), respectively, with $\phi_{0}=\pi / 2$ are plotted here as polar coordinates (i.e. abscissa $=\rho \cos \phi$ and ordinate $=\rho \sin \phi$ ). This represents the trajectory of a particle following the 'radial' geodesic given by equations (47) and (48). The particle starts at $\tau=0$ from $\rho=0$ and moves counterclockwise until it returns to a singular axis $(\rho=0)$ at $\tau=l_{0}$. The return trip takes only about $10 \%$ of the total proper time $l_{0}$.

To determine the energy density of this radiation field measured by a geodesic observer, it is first necessary to consider a solution of the geodesic equation discussed in appendix C. Let us therefore choose a 'radial' geodesic of the free rotating waves given by

$$
\begin{aligned}
& t=\frac{1}{2} l_{0}\left(\zeta^{1 / p}+\zeta^{1 / q}\right), \quad \rho=-\frac{1}{2} l_{0}\left(\zeta^{1 / p}-\zeta^{1 / q}\right), \\
& \phi=\frac{1}{2} \sqrt{5}\left(\zeta^{2 / p}-\zeta^{2 / q}-\sqrt{\frac{3}{2}} \zeta^{4 / 5}\right)+\phi_{0}, \quad z=z_{0},
\end{aligned}
$$

where we have used equations (C7) and (C8) of appendix $\mathrm{C}$ together with the metric in normal form given in section 3, i.e. $\alpha(u)=u / l_{0}^{1 / 2}$ and $\beta(v)=v / l_{0}^{1 / 2}$, and we have fixed the constants in these equations such that $\tau_{1}=\tau_{2}=l_{0}, 2 c_{1}=l_{0}^{1-p / 2}$ and $2 c_{2}=l_{0}^{1-q / 2}$. Note that the scaling parameter $l_{0}$ is therefore related to the intrinsic rotation parameter $l$ via $2 l_{0}=\sqrt{5} l$; moreover, $\phi_{0}$ and $z_{0}$ are constants, $p=4-\sqrt{6}, q=4+\sqrt{6}$ and $\zeta$ is defined by $\zeta=1-\tau / l_{0}$. The observer under consideration here starts at the symmetry axis $\rho=0$, which is regular at $\tau=0$, and returns to it at $\tau=l_{0}$ when it is singular. The trajectory of this observer $\rho(\phi)$ is depicted in figure 3.

Imagine now an orthonormal tetrad frame $\lambda_{(\alpha)}^{\mu}$ that is parallel propagated along this timelike worldline. It is simple to work out explicitly two axes of the tetrad: the time axis and the spatial axis parallel to the symmetry axis. These are given by $\lambda_{(0)}^{\mu}=\mathrm{d} x^{\mu} / \mathrm{d} \tau$ using equations (47) and (48) and $\lambda_{(3)}^{\mu}=\zeta^{-3 / 5} \delta_{3}^{\mu}$, respectively. It follows from the projection of $T_{\mu \nu \rho \sigma}$ on these axes that along the geodesic the gravitational radiation energy density is given by

$$
T_{(0)(0)}=\frac{36}{625 \pi G_{0}} \frac{L^{2}}{\left(l_{0}-\tau\right)^{4}},
$$

while the energy flux along the symmetry axis vanishes, $T_{(0)(3)}=0$, as expected. There is, 
however, radiation pressure along the $z$-axis, which is given by

$$
T_{(3)(3)}=\frac{3}{125 \pi G_{0}} \frac{L^{2}}{\left(l_{0}-\tau\right)^{4}} .
$$

The energy density and the pressure measured by the geodesic observer both diverge at the singularity $\tau=l_{0}$. Note that $T_{(3)(3)} / T_{(0)(0)}=\frac{5}{12}$, which is less than unity as would be expected for the ratio of pressure to density. The characteristic length-scale associated with rotating gravitational waves is $l$; therefore, the constant length $L$ could be chosen to be simply proportional to $l=2 l_{0} / \sqrt{5}$.

\section{Conclusion}

A discussion of the rotating waves is provided starting from a certain ansatz (1) for the spacetime metric. A new class of such spacetimes is found that is given by equations (35) and (31). To make the spacetime metric explicit, a solution of the nonlinear differential equation (31) is required. To find such solutions in general, one must resort to numerical investigations; however, a detailed numerical study of rotating wave spacetimes is beyond the scope of this work. The differential equation (31) has a special exact solution that corresponds to free rotating gravitational waves studied in two previous papers [1,2]; this solution is shown to be locally equivalent to the Siklos solution, which is a special Robinson-Trautman solution.

We present a brief discussion of the properties of the general solution as well as a further treatment of the special solution with particular emphasis on the stress-energy content of the rotating waves. Expressions are provided for the gravitational energy density and the pressure of the radiation parallel to the axis of cylindrical symmetry that are in agreement with physical expectations.

\section{Acknowledgments}

We would like to thank an anonymous referee of a previous version of this paper for pointing out the existence of the additional Killing vector of the solution corresponding to equation (32). This work has been supported by CONACYT, Mexico, grant no 3567-E and DGAPA-UNAM, grant no 121298.

\section{Appendix A. Field equations}

The gravitational field equations for the vacuum region under consideration in this paper are $R_{\mu \nu}=0$. Using the spacetime metric (1) we find

$$
\begin{aligned}
& R_{t t}=\frac{1}{\mu}\left(E_{1}+E_{2}\right)-\mu \omega^{2} \mathrm{e}^{-2 \gamma}\left(E_{1}-E_{2}\right)-\frac{\omega}{\mu} E_{4}-\frac{1}{\mu} E_{7}, \\
& R_{t \rho}=-\frac{\omega}{2 \mu} E_{3}-\frac{1}{\mu} E_{6}, \\
& R_{t \phi}=-\mu \omega \mathrm{e}^{-2 \gamma}\left(E_{1}-E_{2}\right)-\frac{1}{2 \mu} E_{4}, \\
& R_{\rho \rho}=-\frac{1}{\mu}\left(E_{1}+E_{2}-E_{5}\right), \\
& R_{\rho \phi}=-\frac{1}{2 \mu} E_{3},
\end{aligned}
$$




$$
\begin{aligned}
& R_{\phi \phi}=-\mu \mathrm{e}^{-2 \gamma}\left(E_{1}-E_{2}\right), \\
& R_{z z}=\frac{1}{\mu} \mathrm{e}^{-2 \gamma+4 \psi} E_{1},
\end{aligned}
$$

and $R_{t z}=R_{\rho z}=R_{\phi z}=0$. The functions $E_{i}, i=1,2,3, \ldots 7$, are defined by

$$
\begin{aligned}
& E_{1}=\mu\left(\psi_{t t}-\psi_{\rho \rho}\right)+\mu_{t} \psi_{t}-\mu_{\rho} \psi_{\rho} \\
& E_{2}=\mu_{t t}-\mu_{\rho \rho}-\frac{1}{2} \mathrm{e}^{-2 \gamma} \mu^{3} \omega_{\rho}^{2} \\
& E_{3}=\left(\mathrm{e}^{-2 \gamma} \mu^{3} \omega_{\rho}\right)_{t} \\
& E_{4}=\left(\mathrm{e}^{-2 \gamma} \mu^{3} \omega_{\rho}\right)_{\rho} \\
& E_{5}=\mu_{t t}-2 \mu_{\rho \rho}+\mu\left(\gamma_{t t}-\gamma_{\rho \rho}\right)+\mu_{t} \gamma_{t}+\mu_{\rho} \gamma_{\rho}-2 \mu \psi_{\rho}^{2} \\
& E_{6}=\mu_{t \rho}-\left(\mu_{t} \gamma_{\rho}+\mu_{\rho} \gamma_{t}\right)+2 \mu \psi_{t} \psi_{\rho} \\
& E_{7}=2 \mu_{t t}-\mu_{\rho \rho}+\mu\left(\gamma_{t t}-\gamma_{\rho \rho}\right)-\left(\mu_{t} \gamma_{t}+\mu_{\rho} \gamma_{\rho}\right)+2 \mu \psi_{t}^{2}
\end{aligned}
$$

Inspection of the field equations $R_{\mu v}=0$ reveals that these are equivalent to $E_{i}=0, i=$ $1,2,3, \ldots, 7$. In fact, $E_{1}=0$ can be expressed as

$$
\left(\mu \psi_{t}\right)_{t}-\left(\mu \psi_{\rho}\right)_{\rho}=0 \text {. }
$$

Next, equations $E_{3}=0$ and $E_{4}=0$ imply that

$$
\omega_{\rho}=l \mu^{-3} \mathrm{e}^{2 \gamma},
$$

where $l$ is a constant of integration. Using this relation in the equation $E_{2}=0$, we find that

$$
\mu_{t t}-\mu_{\rho \rho}=\frac{1}{2} l^{2} \mu^{-3} \mathrm{e}^{2 \gamma}
$$

Let us now consider the equations $E_{5}=0$ and $E_{7}=0$. Subtracting these equations $\left(E_{7}-E_{5}=0\right)$, we find

$$
\mu_{t t}+\mu_{\rho \rho}=2\left(\mu_{t} \gamma_{t}+\mu_{\rho} \gamma_{\rho}\right)-2 \mu\left(\psi_{t}^{2}+\psi_{\rho}^{2}\right)
$$

Moreover, $E_{7}+E_{5}=0$ can be written as

$$
3\left(\mu_{t t}-\mu_{\rho \rho}\right)+2 \mu\left(\gamma_{t t}-\gamma_{\rho \rho}\right)+2 \mu\left(\psi_{t}^{2}-\psi_{\rho}^{2}\right)=0 ;
$$

however, it turns out that this relation is contained in the rest of the field equations and is therefore redundant. We shall return to this point in the next paragraph. The equation $E_{6}=0$ simply implies that

$$
\mu_{t \rho}=\mu_{t} \gamma_{\rho}+\mu_{\rho} \gamma_{t}-2 \mu \psi_{t} \psi_{\rho}
$$

It can be shown that (A1)-(A5) contain the full content of the gravitational field equations. It proves convenient to express these relations in terms of the null coordinates $u=t-\rho$ and $v=t+\rho$; then, (A1)-(A5) take the form of equations (2)-(6) of section 1 .

It remains to demonstrate that the extra field equation $E_{7}+E_{5}=0$ is indeed redundant. In terms of $u$ and $v$, this equation takes the form $3 \mu_{u v}+2 \mu \gamma_{u v}+2 \mu \psi_{u} \psi_{v}=0$, which using the definition of $W$ in equation (11) can be written as $W=-\mu \psi_{u} \psi_{v}$. Now this relation can be obtained from equations (10), (11) and (2) by substituting $U=\mu \psi_{u}^{2}$ in $\mu U_{v}=\mu_{u} W$ and using (2); alternatively, one can substitute $V=\mu \psi_{v}^{2}$ in $\mu V_{u}=\mu_{v} W$.

Finally, for $l=0$ we find $\omega=\omega(t)$ from (A2) and $\mu_{t t}=\mu_{\rho \rho}$ from (A3). A simple transformation to a rotating frame reduces the waves to non-rotating generalized Beck spacetimes. These have been studied by a number of authors (see [7], ch 15, ch 20 and [15]). 


\section{Appendix B. A special Robinson-Trautman solution}

The purpose of this appendix is to show that the free rotating gravitational wave spacetime is locally the same as a special Robinson-Trautman solution [16]. In the notation of case (ii) in section 2, the metric for the special rotating wave solution, which is algebraically special of Petrov type III, takes the form

$-\mathrm{d} s^{2}=Q^{2}\left(\frac{\mathrm{e}^{-\hat{t}}}{r^{2}} \mathrm{~d} \hat{t}^{2}-\frac{1}{2} \frac{\mathrm{e}^{-\hat{t}}}{r^{4}} \mathrm{~d} r^{2}+\frac{1}{\hat{l}^{2}} \frac{\mathrm{e}^{-\hat{t}}}{r} \mathrm{~d} \Phi^{2}+\frac{2}{\tilde{l}} \frac{\mathrm{e}^{-\hat{t}}}{r^{3 / 2}} \mathrm{~d} \hat{t} \mathrm{~d} \Phi+\mathrm{e}^{\hat{t}} \mathrm{~d} \hat{Z}^{2}\right)$,

where $Q$ is a constant and $\tilde{l}= \pm\left(\frac{3}{2}\right)^{1 / 2} \hat{l}$. Let us recall that the general metric (35) is defined up to an overall constant factor. Here we have restored this factor by introducing the constant $Q$ and set $X(r)= \pm(3 r / 2)^{-1 / 2}$ for the special solution (32). The metric (B1) is invariant under a three-parameter group of isometries characterized by the spacelike Killing vectors

$\boldsymbol{X}_{1}=-\frac{4}{3}\left(-2 \partial_{\hat{t}}+r \partial_{r}-\frac{1}{2} \Phi \partial_{\Phi}+\hat{Z} \partial_{\hat{Z}}\right), \quad \boldsymbol{X}_{2}=\partial_{\Phi}+\partial_{\hat{Z}}, \quad \boldsymbol{X}_{3}=\partial_{\Phi}-\partial_{\hat{Z}}$.

The Bianchi type is determined by the structure constants of the Lie algebra of the group $G_{3}$ under consideration here. This can be expressed in normal form as

$\left[\boldsymbol{X}_{1}, \boldsymbol{X}_{2}\right]=-\boldsymbol{X}_{3}+\frac{1}{3} \boldsymbol{X}_{2}, \quad\left[\boldsymbol{X}_{2}, \boldsymbol{X}_{3}\right]=0, \quad\left[\boldsymbol{X}_{3}, \boldsymbol{X}_{1}\right]=\boldsymbol{X}_{2}-\frac{1}{3} \boldsymbol{X}_{3}$,

which therefore represents a type $\mathrm{VI}_{h}$ with $h=-\frac{1}{9}$ in the Bianchi classification [7].

We now proceed to show that our special solution is locally equivalent to a solution that belongs to the same Bianchi and Petrov types and is a special Robinson-Trautman solution that can be written as (see equation (5.19) of [17] and equation (33.1) on p 378 of [7])

$$
-\mathrm{d} s^{2}=\frac{\hat{r}^{2}}{\hat{x}^{3}}\left(\mathrm{~d} \hat{x}^{2}+\mathrm{d} \hat{y}^{2}\right)-2 \mathrm{~d} \hat{u} \mathrm{~d} \hat{r}+\frac{3}{2} \hat{x} \mathrm{~d} \hat{u}^{2} .
$$

It can be shown that this is the only algebraically special vacuum solution with diverging rays and a maximal $G_{3}$ [17]. Moreover, it has been shown by Siklos in 1978 (as referred to in [7]) that it represents a hypersurface-homogeneous spacetime (see section 11.3.2 of [7]). By studying the invariants of the Killing vectors in (B1) and (B2), etc, we arrive at the coordinate transformation $(\hat{t}, r, \Phi, \hat{Z}) \rightarrow(\hat{r}, \hat{x}, \hat{u}, \hat{y})$ with

$$
\mathrm{e}^{\hat{t}}=\tilde{l}^{-2} \frac{\hat{r}^{2}}{\hat{x}^{3}}, \quad r=\tilde{l}^{2} \frac{\hat{x}^{2}}{\hat{r}^{2}}, \quad \Phi=\hat{u}-2 \frac{\hat{r}}{\hat{x}}, \quad \hat{Z}=\hat{y} .
$$

Under this transformation (B1) can be written as

$$
-\mathrm{d} s^{2}=\left(\frac{Q}{\tilde{l}}\right)^{2}\left[\frac{\hat{r}^{2}}{\hat{x}^{3}}\left(\mathrm{~d} \hat{x}^{2}+\mathrm{d} \hat{y}^{2}\right)-2 \mathrm{~d} \hat{u} \mathrm{~d} \hat{r}+\frac{3}{2} \hat{x} \mathrm{~d} \hat{u}^{2}\right],
$$

which with $(Q / \tilde{l}) \hat{r} \rightarrow \hat{r}$ and $(Q / \tilde{l}) \hat{u} \rightarrow \hat{u}$ reduces to the metric (B2). Therefore, the special rotating wave solution (B1) described in section 3 and the special Robinson-Trautman solution (B2) are locally equivalent. The fact that the rays are non-twisting in any Robinson-Trautman solution is not in conflict with our interpretation of this solution in terms of rotating gravitational waves, since this refers to the waves that are moving radially as well as azimuthally.

\section{Appendix C. Geodesics in rotating wave spacetimes}

Starting with equation (1), let

$\mathcal{L}_{g}=-\frac{1}{2}\left(\frac{\mathrm{d} s}{\mathrm{~d} \lambda}\right)^{2}=\frac{1}{2}\left[\mathrm{e}^{2 \gamma-2 \psi}\left(-\dot{t}^{2}+\dot{\rho}^{2}\right)+\mu^{2} \mathrm{e}^{-2 \psi}(\omega \dot{t}+\dot{\phi})^{2}+\mathrm{e}^{2 \psi} \dot{z}^{2}\right]$ 
where $\dot{t}=\mathrm{d} t / \mathrm{d} \lambda$, etc. Since this Lagrangian does not depend upon $\phi$ and $z$, there are two constants of the motion $p_{\phi}$ and $p_{z}$ given by

$$
p_{\phi}=\frac{\partial \mathcal{L}_{g}}{\partial \dot{\phi}}=\mu^{2} \mathrm{e}^{-2 \psi}(\omega \dot{t}+\dot{\phi}), \quad p_{z}=\frac{\partial \mathcal{L}_{g}}{\partial \dot{z}}=\mathrm{e}^{2 \psi} \dot{z} .
$$

Moreover, we have

$$
\frac{\mathrm{d}}{\mathrm{d} \lambda}\left(\omega p_{\phi}-\mathrm{e}^{2 \gamma-2 \psi} \dot{t}\right)=\frac{\partial \mathcal{L}_{g}}{\partial t}, \quad \frac{\mathrm{d}}{\mathrm{d} \lambda}\left(\mathrm{e}^{2 \gamma-2 \psi} \dot{\rho}\right)=\frac{\partial \mathcal{L}_{g}}{\partial \rho} .
$$

Let us now focus attention on 'radial' geodesics such that the momenta associated with azimuthal and vertical motions vanish. It is then convenient to write the equations of motion in terms of radiation coordinates $u$ and $v$. We find that equations (C2) and (C3) reduce to

$\frac{\mathrm{d}}{\mathrm{d} \lambda}\left(\mathrm{e}^{2 \gamma-2 \psi} \frac{\mathrm{d} u}{\mathrm{~d} \lambda}\right)=\left(\mathrm{e}^{2 \gamma-2 \psi}\right)_{v} \frac{\mathrm{d} u}{\mathrm{~d} \lambda} \frac{\mathrm{d} v}{\mathrm{~d} \lambda}, \quad \frac{\mathrm{d}}{\mathrm{d} \lambda}\left(\mathrm{e}^{2 \gamma-2 \psi} \frac{\mathrm{d} v}{\mathrm{~d} \lambda}\right)=\left(\mathrm{e}^{2 \gamma-2 \psi}\right)_{u} \frac{\mathrm{d} u}{\mathrm{~d} \lambda} \frac{\mathrm{d} v}{\mathrm{~d} \lambda}$,

which imply that

$$
\mathrm{e}^{2 \gamma-2 \psi} \frac{\mathrm{d} u}{\mathrm{~d} \lambda} \frac{\mathrm{d} v}{\mathrm{~d} \lambda}
$$

is a constant along the path. This constant vanishes for a null geodesic, so that 'radial' null geodesics correspond to $u=t-\rho=$ constant or $v=t+\rho=$ constant, where $\lambda$ is the affine parameter along the path.

We are mainly interested in timelike 'radial' geodesics, hence we set $\lambda=\tau$, where $\tau$ is the proper time along the path. Then, with $\tilde{U}=\mathrm{d} u / \mathrm{d} \tau$ and $\tilde{V}=\mathrm{d} v / \mathrm{d} \tau$, we have $\tilde{U} \tilde{V}=\mathrm{e}^{2 \psi-2 \gamma}$. It follows from (C4) that $\tilde{U}_{v} \tilde{V}^{2}=\tilde{V}_{u} \tilde{U}^{2}$. The general solution is given by

$$
\frac{\mathrm{d} u}{\mathrm{~d} \tau}=\frac{1}{2}\left(\frac{\partial \mathcal{S}}{\partial u}\right)^{-1}, \quad \frac{\mathrm{d} v}{\mathrm{~d} \tau}=\frac{1}{2}\left(\frac{\partial \mathcal{S}}{\partial v}\right)^{-1},
$$

where $\mathcal{S}(u, v)$ is any solution of the differential equation

$$
4 \frac{\partial \mathcal{S}}{\partial u} \frac{\partial \mathcal{S}}{\partial v}=\mathrm{e}^{2 \gamma-2 \psi}
$$

This is, in fact, the Hamilton-Jacobi equation for 'radial' timelike geodesics, and $\mathcal{S}$ along the path differs from the proper time by a constant. We now consider the special solution corresponding to free waves discussed in section 3 . In this case, it is simple to illustrate a class of solutions of the eikonal equation (C6) via separation of variables, i.e.

$$
\mathcal{S}(u, v)=-c_{1} \alpha^{p}(u)-c_{2} \beta^{q}(v),
$$

where $p=4-\sqrt{6}, q=4+\sqrt{6}$, and $c_{1}$ and $c_{2}$ are constants such that $c_{1} c_{2}=1 / 5 l^{2}$. Equations (C5) now have solutions

$$
\alpha(u)=\left(\frac{\tau_{1}-\tau}{2 c_{1}}\right)^{1 / p}, \quad \beta(v)=\left(\frac{\tau_{2}-\tau}{2 c_{2}}\right)^{1 / q},
$$

where $\tau_{1}$ and $\tau_{2}$ are constants of integration. More explicitly, let us consider the normal form of the metric with $\alpha(u)=l_{0}^{-1 / 2} u$ and $\beta(v)=l_{0}^{-1 / 2} v$ as employed in section 4. The geodesic would then hit the null singular hypersurface $u=0$ (or $v=0$ ) at $\tau=\tau_{1}$ (or $\tau=\tau_{2}$ ). The singular nature of these moving cylinders can be seen from the fact that the geodesic cannot be continued past them since then $u$ or $v$ would become complex. 


\section{References}

[1] Mashhoon B and Quevedo H 1990 Phys. Lett. A 151464

[2] Quevedo H and Mashhoon B 1991 Relativity and Gravitation: Classical and Quantum, Proc. 7th Conf. SILARG ed J C D’Olivo et al (Singapore: World Scientific) pp 287-93

[3] Ardavan H 1984 Phys. Rev. D 29207

Ardavan H 1989 Proc. R. Soc. A 424113

[4] Ardavan H 1984 Classical General Relativity ed W B Bonnor, J N Islam and M A H MacCallum (Cambridge: Cambridge University Press) pp 5-14

[5] Gaffet B 1990 Class. Quantum Grav. 72017

[6] Bel L 1962 Colloques Internationaux du CNRS (Paris) pp 119-26

Robinson I 1962 Colloques Internationaux du CNRS (Paris) pp 119-26

See also Mashhoon B, McClune J C and Quevedo H 1997 Phys. Lett. A 23147

Mashhoon B, McClune J C and Quevedo H 1999 Class. Quantum Grav. 161137

[7] Kramer D, Stephani H, MacCallum M and Herlt E 1980 Exact Solutions of Einstein's Field Equations (Cambridge: Cambridge University Press)

[8] Zakharov V D 1973 Gravitational Waves in Einstein's Theory (New York: Halsted) (translated by R N Sen from the 1972 Russian edition)

[9] Mars M and Senovilla J M M 1993 Class. Quantum Grav. 101633

Mars M and Senovilla J M M 1995 Class. Quantum Grav. 122071

MacCallum M A H and Santos N O 1998 Class. Quantum Grav. 151627

[10] Beck G 1925 Z. Phys. 33713

[11] Einstein A and Rosen N 1937 J. Franklin Inst. 22343

Rosen N 1954 Bull. Res. Council Israel 3328

[12] Bonnor W B 1957 J. Math. Mech. 6203

Weber J and Wheeler J A 1957 Rev. Mod. Phys. 29509

[13] Van den Bergh N and Wils P 1986 Class. Quantum Grav. 2229

[14] Bel L 1958 C. R. Acad. Sci. Paris 2471094

Bel L 1959 C. R. Acad. Sci. Paris 2481297

Bel L 1962 Cah. Phys. 1659

Debever R 1958 Bull. Soc. Math. Belg. 10112

[15] Verdaguer E 1993 Phys. Rep. 2291

Griffiths J B 1991 Colliding Plane Waves in General Relativity (Oxford: Oxford University Press)

Alekseev G A and Griffiths J B 1996 Class. Quantum Grav. 132191

[16] Robinson I and Trautman A 1962 Proc. R. Soc. A 265463

[17] Kerr R P and Debney G C 1970 J. Math. Phys. 112807 